\title{
CUIDADOS DE ENFERMAGEM COM PRESSÃO ARTERIAL DO PACIENTE SUBMETIDO À CIRURGIA CARDÍACA COM CIRCULAÇÃO EXTRACORPÓREA
}

\section{ARTIGO ORIGINAL}

OLIVEIRA, Joana de Oliveira ${ }^{1}$, SILVA, Ysa Mara Lourenço ${ }^{2}$, SILVA, Carlos Magno Carvalho da ${ }^{3}$, CARVALHO, Damaris Braga Vieira ${ }^{4}$, REIS, Maciane ${ }^{5}$

OLIVEIRA, Joana de Oliveira. Et al. Cuidados De Enfermagem Com Pressão Arterial Do Paciente Submetido À Cirurgia Cardíaca Com Circulação Extracorpórea. Revista Científica Multidisciplinar Núcleo do Conhecimento. Ano 06, Ed. 06, Vol. 17, pp. 156-166. Junho de 2021. ISSN: 2448-0959, Link de acesso: https://www.nucleodoconhecimento.com.br/saude/paciente-submetido, DOI: 10.32749/nucleodoconhecimento.com.br/saude/paciente-submetido

\section{RESUMO}

A cirurgia cardíaca ainda é uma das intervenções para as doenças arteriais coronarianas. Após esta intervenção, o paciente inicia o pós- operatório com os

\footnotetext{
${ }^{1}$ Enfermeira Pós-graduada em Enfermagem cardiovascular e hemodinâmica. Bacharela em enfermagem pelo centro universitário Jorge Amado- BA. Especialização em enfermagem em Cardiologia e HemodinâmicaAtualiza Cursos- BA. Especialização em enfermagem nos moldes de Residência: clínica médica e cirurgia- GeralUnirio.

${ }^{2}$ Formada em Enfermagem pela Faculdade Novo Milênio, Vila Velha - Espírito Santo, Bacharela em fisioterapia da Universidade Veiga de Almeida, Rio de Janeiro. Especialista nos moldes da residência em enfermagem e cirurgia geral.

${ }^{3}$ Professor do departamento de Enfermagem médico-cirúrgica da escola de Enfermagem Alfredo Pinto (EEAP). Professor de pós- graduação da escola de Enfermagem Alfredo Pinto (stricto e latu sensu). Residência em Enfermagem. Mestrado na Universidade Federal Fluminense (UFF) e doutorado na Universidade Federal do Estado do Rio de Janeiro (UFRJ). Doutor pela UFRJ. Bacharel em Enfermagem.

${ }^{4}$ Doutoranda em Enfermagem pela Universidade Federal do Rio de Janeiro. Mestre em Enfermagem pela Universidade Federal do Rio de Janeiro. Especialização em Circulação Extracorpórea e Assistência Circulatória Mecânica pelo Instituto do Coração do Hospital das Clínicas da Faculdade de Medicina da Universidade de São Paulo. Especialização em Cardiologia pela Universidade Gama Filho. Graduação em Enfermagem pela Universidade do Grande Rio Professor José de Souza Herdy.

${ }^{5}$ Mestre em enfermagem pela UFRJ - departamento médico-cirúrgico / Saúde Mental; Graduada em enfermagem pela UERJ.
}

RC: 90178

Disponível em: https://www.nucleodoconhecimento.com.br/saude/paciente-submetido 
valores pressóricos alterados, necessitando de cuidados intensivos. Objetivo: analisar as variações da pressão arterial durante o pós-operatório (PO) do paciente submetido a cirurgia cardíaca com circulação extracorpórea (CEC) e discutir os cuidados de enfermagem. Metodologia: Trata-se de uma pesquisa documental, de abordagem qualitativa e descritiva. A instituição da pesquisa foi um Hospital Federal de caráter militar, que tem a sede no município do Rio de Janeiro. A coleta de dados ocorreu no mês de abril de 2018, com amostra de 30 prontuários de pacientes submetidos à cirurgia cardíaca com circulação extracorpórea durante o período de janeiro à julho de 2017, através de uma ficha de pós- operatório das anotações da equipe de enfermagem. Resultado: Quanto ao perfil, a maioria tinha idade maior que sessenta e um anos, o gênero masculino apareceu em maior escala com $77 \%$ ( $n=23)$, em relação ao gênero feminino com $23 \%(n=07)$. Os pacientes mantiveram os valores da PAM (pressão arterial média) entre 70 a $110 \mathrm{mmHg}$ que são considerados normais, destes $83,3 \%(n=25) 70$ a $100 \mathrm{mmHg}$, normotensos; $13,3 \%(n=4)<70 \mathrm{mmHg}$, hipotensos; $3,47 \%(n=1)>110 \mathrm{mmHg}$ na primeira hora mantiveram hipertensos. Conclusão: A recuperação do paciente que foi submetido a cirurgia cardíaca depende de um (POI) pós-operatório imediato eficaz, e posteriormente do PO, desta forma o enfermeiro deve acompanhar a monitorização e prestar uma assistência especializada para este paciente crítico.

Palavras-chave: Cirurgia torácica, Cuidados de enfermagem, Pressão Arterial, Período pós-operatório.

\section{INTRODUÇÃO}

A cirurgia cardíaca, é considerado um procedimento de alto risco. Assim, é essencial que o pós-operatório do paciente, seja realizado de forma correta, a fim de evitar complicações. O pós-cirúrgico quando bem executado, colabora com o aumento na qualidade de vida e redução dos índices de possíveis doenças relacionadas. (SANTANA et. al., 2013). 
Quando o paciente é admitido na Unidade Coronariana/ UTI cardíaca, é necessária uma avaliação do enfermeiro para assegurar a estabilidade. O cuidado com a monitorização contínua, medida da pressão arterial pelo cateter arterial, medida da pressão arterial pulmonar, parâmetros hemodinâmicos derivados, como pós-carga e débito cardíaco e índices de contratilidade, são essenciais nesse caso. (TORRATI e DANTAS, 2012).

Diante das afirmativas anteriores, questiona-se qual é a importância de o enfermeiro realizar uma avaliação hemodinâmica do paciente submetido a cirurgia cardíaca no pós-operatório imediato e quais cuidados são necessários para manter o paciente com os níveis pressóricos adequados.

Neste sentido, o levantamento dos documentos do prontuário do paciente é uma fonte rica de dados, o que servirá de método para este artigo. Espera-se que os resultados desta pesquisa, realizada no Hospital Federal de caráter militar, tragam implicações e melhorias nos cuidados dos pacientes Trata-se de uma pesquisa documental, de abordagem qualitativa e descritiva. Assim, este estudo tem por objetivo, analisar as variações da pressão arterial durante o pós- operatório do paciente submetido a cirurgia cardíaca com Circulação extracorpórea e discutir os cuidados de enfermagem dentro desse quadro.

\section{DESENVOLVIMENTO}

O paciente que foi submetido à cirurgia cardíaca, deve ser acompanhado de forma sistemática pela observação contínua, pelo traçado eletrocardiográfico e análise periódica de vários parâmetros hemodinâmicos no pós-operatório. A hipertensão arterial ocorre em $48 \%$ a $55 \%$ dos pacientes durante as primeiras quatro a seis horas após a cirurgia cardíaca, sendo que durante as primeiras doze horas após a cirurgia, poderá ocorrer a hipotensão.(WOODS, 2005).

Manter uma pressão arterial satisfatória, no paciente do pós-operatório, implica em preservar a cirurgia pois a hipertensão arterial gera um aumento na pré-carga, 
arritmias e disfunção miocárdica, sangramentos e pode ocorrer rutura nas linhas de sutura; assim também como a hipotensão deve ser corrigida de imediato, prevenindo estado de choque, infarto agudo do miocárdico, arritmias e outras possíveis complicações. Diante dessas observações surge a inquietação para saber se a pressão arterial dos pacientes está controlada durante o período pós- operatório. (DUARTE et. al., 2012).

Segundo dados do DATASUS (2012), a taxa internação por doenças isquêmicas do coração em 2012 foi de 242.858 no Brasil. Entre elas incluem-se as complicações atuais subsequentes ao infarto agudo do miocárdio, doenças isquêmicas agudas do coração e doença isquêmica crônica do coração, que pode levar o paciente para uma intervenção de Cirurgia Cardíaca. (BRASIL, 2012; PERGHER e SILVA, 2014).

A cirurgia cardíaca ainda é um tratamento sugerido, para alguns casos de cardiopatias. É considerada cirurgia de grande porte e, na maioria das vezes, é utilizada à circulação extracorpórea, causando distúrbios hidroeletrolíticos e instabilidade hemodinâmica. Isso reforça a necessidade de cuidados intensivos no pós-operatório imediato. (DESSOTTE et. al, 2016).

De acordo com Torrati e Dantas (2012), a circulação extracorpórea (CEC), é um procedimento muito utilizado, cuja finalidade é preservar as características funcionais do coração e oferecer segurança à equipe cirúrgica. É uma técnica-padrão, na qual ela realiza de uma forma mecânica o trabalho do coração e pulmão; apesar do risco eminente de complicações neurológicas, a opção da CEC ainda é a preferência, dadas as evidências de viabilidade e segurança. As complicações pós-operatórias de cirurgias cardíacas, estão relacionadas também ao grau de risco dos pacientes coronariopatas, e não apenas à utilização da circulação extracorpórea.(GODINHO et. al., 2012)

No momento do perioperatório, os valores da pressão arterial podem alterar. Assim, será necessário intervenção imediata, uma vez que ocorre instabilidade hemodinâmica. Essa instabilidade se dá principalmente relacionada à pressão arterial, 
onde fatores como indução anestésica, ventilação mecânica, circulação extracorpórea, uso de drogas, dor, estresse físico e emocional, estão envolvidos. Por este motivo o paciente já chega no pós- operatório necessitando destes cuidados intensivos com os valores pressóricos alterados. (PONTE et. al., 2010).

Por se tratar de um período de cuidado crítico, o pós- operatório imediato (POI) de cirurgias cardíacas, momento o qual se observa e se assiste a recuperação do paciente em pós- anestésico e em pós- estresse cirúrgico, é marcado pela instabilidade do quadro clínico do paciente. (DUARTE et. al., 2012)

A hipertensão Arterial, é uma situação comum no pós-operatório de cirurgia cardíaca. É comumente causada pela estimulação do sistema simpático por meio dos níveis elevados de noradrenalina, renina- angiotensina e vasopressina, associados a circulação extracorpórea.). Enquanto a hipotensão arterial pode levar à hipoperfusão de órgãos vitais e ocasionar danos isquêmicos importantes. (AULER et. al., 2004; PONTE et. al., 2010).

Após a cirurgia cardíaca, o paciente é admitido em uma unidade de cuidado intensivo para monitorização rigorosa por 12 a 24 horas após a cirurgia. O cuidado e a rotina incluem: monitorização contínua de ECG, medida de pressão arterial pelo cateter arterial, oximetria de pulso, medida da temperatura corporal, pressão arterial pulmonar para estimular a pré-carga e medida do débito cardíaco, bem como cálculo de parâmetros hemodinâmicos (como pós-carga, débito cardíaco e índices de contratilidade). (WOODS, 2005).

\section{RESULTADOS E DISCUSSÃO}

A instituição onde o estudo foi realizado, foi um Hospital Federal, de caráter militar, com sede no município do Rio de janeiro, que teve como parecer técnico favorável ao projeto de pesquisa: Cuidados de Enfermagem com a Pressão Arterial do Paciente Submetido à Cirurgia Cardíaca com Circulação Extracorpórea. A escolha do local se deu pela inserção das pesquisadoras no hospital, na qual cursava Residência de 
enfermagem. Cada coleta recebeu uma numeração para que informações coletadas nos prontuários, mantivessem a confidencialidade e a privacidade dos pacientes submetidos à cirurgia cardíaca.

A coleta de dados ocorreu no mês de abril de 2018, com prontuários de pacientes submetidos à cirurgia cardíaca com circulação extracorpórea durante o período de janeiro a julho de 2017, através de uma ficha de pós- operatório das anotações da equipe de enfermagem. Os prontuários foram disponibilizados pelo Serviço de Arquivo Médico e a partir do levantamento dos registros de enfermagem, foi possível coletar as anotações dos cuidados com a pressão arterial invasiva que ocorreram nas primeiras seis horas. Após selecionada a amostra documental e leitura compreensiva do material, foi realizada a análise e discussão. Os dados obtidos foram catalogados no programa exce/por meio de gráficos e abordagem descritiva.

Foram analisados 30 prontuários, no período de janeiro a julho de $2017 . \mathrm{Na}$ caracterização dos sujeitos, o gênero masculino apareceu em maior escala com $77 \%$ $(n=23)$, em relação ao gênero feminino com $23 \%(n=07)$. Em relação à idade, foram consideradas idosas as pessoas com idade igual ou superior a 61 anos $(n=24)$ e os adultos participantes do estudo tem idades entre 40 e 60 anos $(n=06)$.

GRÁFICO 1 Tipos de Cirurgias Cardíacas

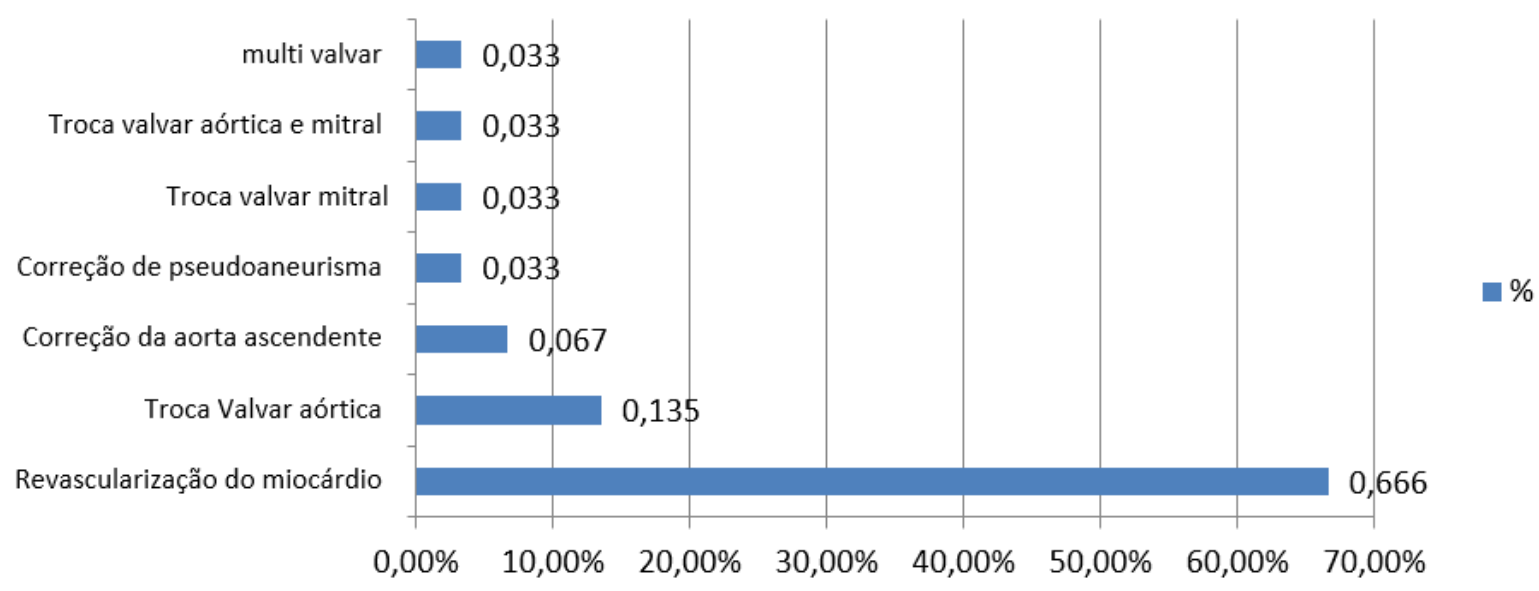

Fonte: Os autores. 
Os pacientes submetidos à cirurgia mantiveram os valores da PAM (pressão arterial média) entre 70 à $110 \mathrm{mmHg}$, que são considerados normais. Destes, 83,3\% ( $\mathrm{n}=25)$ 70 a $100 \mathrm{mmHg}$, normotensos; $13,3 \%(n=4)<70 \mathrm{mmHg}$, hipotensos; $3,47 \%(n=1)$ $>110 \mathrm{mmHg}$, onde na primeira hora mantiveram hipertensos, respectivamente.

Para Pergher e Silva (2014), no pós-operatório de cirurgia cardíaca não é recomendável manter hipotensão, hipertensão ou variações bruscas de pressão arterial. Desta forma, torna-se necessário a monitorização da PAM de forma rigorosa. A PAM, é considerada um dos parâmetros essenciais do paciente crítico, pois proporciona contínua mensuração das pressões arteriais sistólica, média e diastólica. A média das 6 primeiras horas mantiveram dentro do padrão da normalidade 96,6\% $(n=29)$ normotensos e apenas $3,4 \%(n=1)$, manteve a PAM abaixo de $70 \mathrm{mmHg}$. Observa-se que os 20 pacientes da Revascularização do miocárdio mantiveram a média da PAM entre $78,9 \mathrm{mmHg}$ nas primeiras 6 horas. A seguir, serão descritos os medicamentos que foram utilizados no POI. (SHIMOYA-BITTENCOURT et. al., 2014).

Os medicamentos utilizados para manter os níveis pressóricos foram: 
GRÁFICO 2 Medicação utilizada para manutenção dos níveis pressóricos

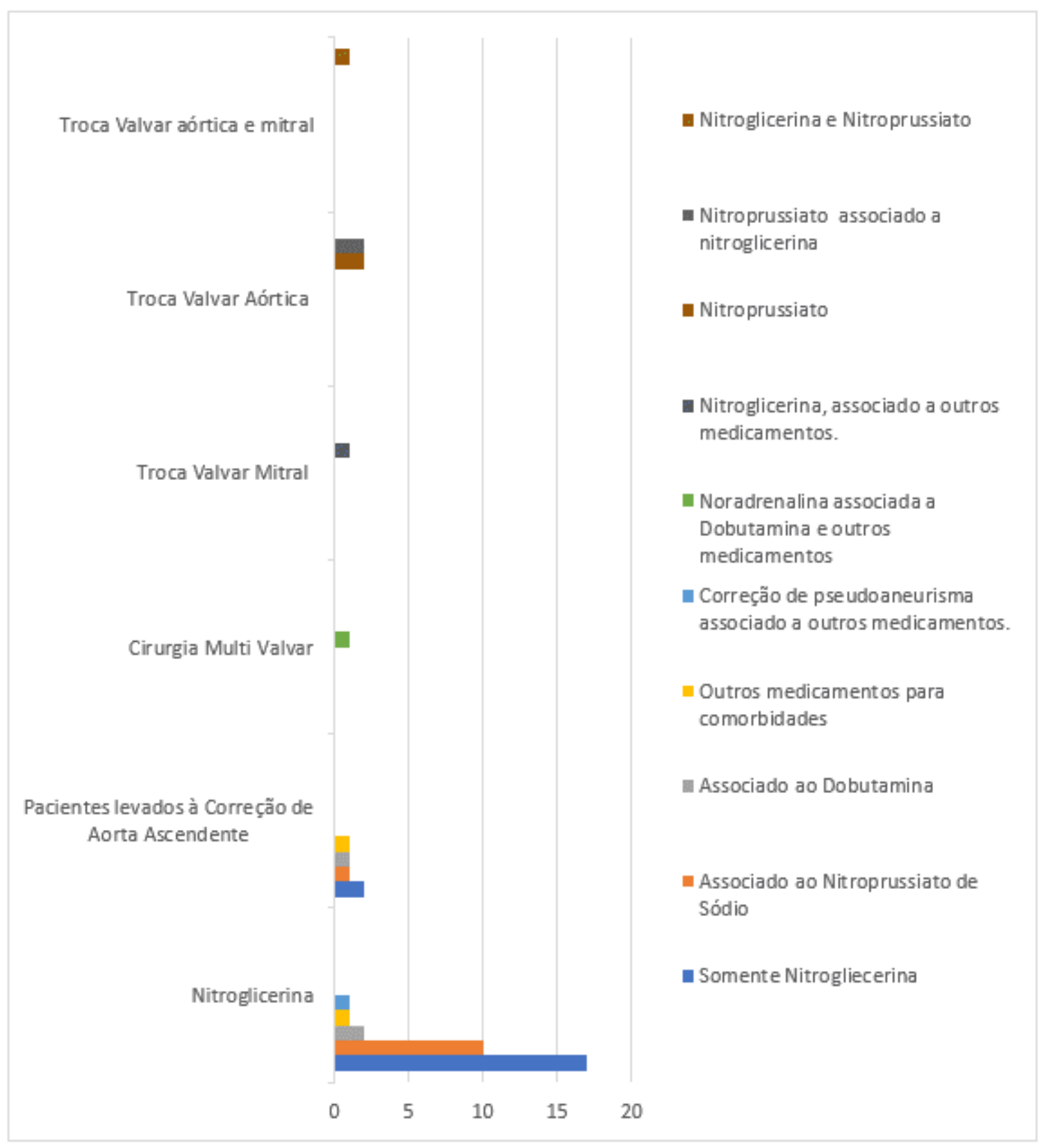

Fonte: Os autores.

Referente aos resultados do gráfico:

- Dezessete pacientes com nitroglicerina e destes dez pacientes utilizaram associado o nitroprussiato de sódio. Apenas dois pacientes utilizaram Dobutamina.

RC: 90178

Disponível em: https://www.nucleodoconhecimento.com.br/saude/paciente-submetido 
- Nos registros das 6 horas não foi encontrado uso de noradrenalina e amiodarona.

- Dos dois pacientes que foram submetidos à Correção de aorta ascendente, um utilizou nitroprussiato de sódio e o outro utilizou Dobutamina, além de outros medicamentos para comorbidades.

- Correção de pseudoaneurisma utilizou nitroglicerina, apenas um paciente foi submetido a esta cirurgia, também associado a outros medicamentos.

- Cirurgia multi valvar apenas um paciente foi submetido, utilizou noradrenalina associada a dobutamina e outros medicamentos.

- Troca valvar mitral um paciente foi utilizado nitroglicerina, associado a outros medicamentos. Dos quatro pacientes que fizeram a cirurgia de troca Valvar Aórtica foi utilizado nitroprussiato e destes dois foi necessário associar a nitroglicerina.

- Somente um paciente foi submetido à cirurgia de troca Valvar aórtica e mitral, e utilizou-se nitroglicerina e nitroprussiato.

Observa- se na ficha do pós- operatório, que todos os pacientes utilizaram solução para reposição de eletrólitos ( $2 \mathrm{~g}$ de magnésio e $4 \mathrm{~g}$ de potássio com $440 \mathrm{ml}$ S.F 0,9\%). A reposição de potássio é frequentemente necessária, devido a perda de sangue na cirurgia com circulação extracorpórea.

Estudos comprovam que predominância das cirurgias cardíacas é do sexo masculino, sendo confirmado através do resultado: $77 \%$ homens. Na ficha de enfermagem do pós- operatório, observou-se que a maioria dos pacientes (77\%) eram hipertensos.

Neste sentido, a enfermagem desempenha um papel importante no POI. Os cuidados de enfermagem foram realizados de acordo com o horário da chegada do paciente na Unidade Coronariana (UC) e rotinas do setor, as quais são baseadas nos protocolos e checklist de pós- operatório. O leito para a acomodação do paciente encontra-se preparado com o nome do paciente e tipo de cirurgia que foi realizada; é checada a prescrição médica para iniciar os primeiros cuidados do $\mathrm{POI}$. 
$\mathrm{Na}$ avaliação da admissão, o enfermeiro observa a PAM e o ritmo cardíaco, checa todos os cateteres, os tipos de infusões, os drenos de mediastino, pois no momento do transporte do Centro Cirúrgico para a Unidade Coronariana pode ocorrer deslocamento ou tracionamento dos tubos ou cateteres, causando instabilidade hemodinâmica.

No monitor é estabelecido os limites de alarme, os cateteres intravasculares são conectados ao monitor e observa-se os traçados da curva para saber se realmente a PAM no paciente está funcionante; zera, calibra o sistema e documenta todos os procedimentos e assistência. Pressões hemodinâmicas exatas são essenciais para a avaliação precisa e tratamento de complicações pós-operatórias.

O enfermeiro fica na beira leito observando oscilações da PAM. A hipertensão está associada a resistência vascular e níveis elevados de renina e catecolaminas plasmáticas no pós- operatório da cirurgia Cardíaca. A HAS - Hipertensão Arterial Sistemática, também aumenta a tensão da parede aórtica, aumentando desta forma a demanda de oxigênio miocárdico, podendo suceder a isquemia miocárdica, podendo aumentar as margens de sutura aórtica e sangramentos, o que é evitado no POI. A hipotensão pode ocorrer nas primeiras 12 horas e deve ser corrigida de imediato, prevenindo estado de choque, infarto agudo do miocárdio, arritmias e outras possíveis complicações. Avaliar os exames laboratoriais de base é essencial, o enfermeiro realiza a coleta de sangue e verifica as gasometrias arterial e venosa para acompanhar o estado fluido, eletrolítico e respiratório.

Para as anotações da assistência utiliza-se a ficha específica "Pós- operatório de Cirurgia cardíaca" que é de responsabilidade do enfermeiro preencher, contendo as informações de parâmetros hemodinâmicos, diagnóstico de internação, drogas utilizadas, alergia medicamentosa, tipos de drenos e dispositivos invasivos.

\section{CONSIDERAÇÔES FINAIS}


A revascularização do miocárdio com circulação extracorpórea foi a cirurgia mais realizada devido a maioria dos pacientes terem como diagnóstico a doença arterial coronariana, mesmo com o avanço dos procedimentos menos invasivos. A PAM nas primeiras seis horas do pós-operatório foi mantida nos níveis de normalidade da maioria dos pacientes.

Devido ao controle hemodinâmico, controle da dor, sangramento do mediastino mínimo e ausência de complicações neurológicas foi possível a extubação antes das seis primeiras horas. A recuperação do paciente que foi submetido à cirurgia cardíaca depende de um POI - Pós-Operatório Imediato eficaz, e posteriormente do PO - Pósoperatório, desta forma o enfermeiro deve recebê-lo na entrada da Unidade Coronariana/ UTI.

O cuidado deve ser individualizado, visando manter o equilíbrio hemodinâmico nas primeiras $24 \mathrm{~h}$, a vigilância no monitor, eletrocardiograma, saturação de oxigênio, pressões derivadas de cateteres invasivos, além da avaliação dos débitos dos drenos torácicos e volume de diurese, que são imprescindíveis neste contexto. Esses cuidados ajudam a reduzir o tempo de internação do paciente.

Conclui-se que, diante do paciente crítico, o enfermeiro deve ter conhecimento técnico-científico aplicando ainda uma assistência humanizada. Assim será garantido a dignidade do ser humano, englobando os direitos do paciente, sua subjetividade e seus aspectos culturais, promovendo um cuidado de excelência. Devido a todo o processo que o paciente enfrentou desde o diagnóstico até o momento da cirurgia, faz-se necessário, além dos cuidados de enfermagem, uma equipe multidisciplinar e acompanhamento por longo período para reestabelecer a qualidade de vida do paciente.

\section{REFERÊNCIAS}


AULER, José Otávio; et.al. Pós-Operatório de Cirurgia Torácica e Cardiovascular.5 ed. Porto Alegre: ed. Artmed. 2004. p.225-228. Disponível em DOI: https://doi.org/10.1590/S0102-76382004000300005

BRASIL. Ministério da Saúde. Departamento de informática do sistema único de saúde/DATASUS. Sistema de Informações Hospitalares do SUS - SIH/SUS. IBGE: base demográfica. 2012.2 Disponível http://www2.datasus.gov.br/DATASUS/index.php?area=0201\&id=1421686 Acesso em: 07 de junho de 2021.

DESSOTE, Carina Aparecida; et.al. Estressores percebidos por pacientes no pósoperatório imediato de cirurgia cardíaca. Revista Brasileira de Enfermagem. 2016. p.741-750. Disponível em DOI: http://dx.doi.org/10.1590/0034-7167.2016690418i Acesso em: 07 de junho de 2021.

DUARTE, Sabrina; et.al. 0 cuidado de enfermagem no pós-operatório de cirurgia cardíaca: um estudo de caso. . Escola Anna Nery. Rio de Janeiro. Out-dez 2012. Disponível em: https://doi.org/10.1590/S1414-81452012000400003 Acesso em: 07 de junho de 2021.

GODINHO Ana Sofia; et.al. Cirurgia de revascularização miocárdica com circulação extracorpórea versus sem circulação extracorpórea: uma metanálise. Arquivos Brasileiros de Cardiologia. 2012.p.87-94. Disponível em: https://doi.org/10.1590/S0066-782X2012000100014 Acesso em: 06 de junho de 2021.

PERGHER, Adele; SILVA, Roberto Carlos. Tempo estímulo-resposta aos alarmes de pressão arterial invasiva: implicações para a segurança do paciente crítico. Revista Gaúcha de Enfermagem. 2014. On-line. Disponível em: https://doi.org/10.1590/1983-1447.2014.02.43715 Acesso em: 07 de junho de 2021.

PONTE, Keila Maria; et.al. Controle Pressórico de Pacientes Submetidos à Cirurgia Cardíaca. Revista da Rede de Enfermagem Fortaleza, v. 11, n. 4. Out./dez.2010. 118-126.

Disponível em: 
http://www.repositorio.ufc.br/bitstream/riufc/4416/1/2010_art_mamsilva1.pdf Acesso em: 07 de junho de 2021.

SANTANA, Geiza; et.al. Avaliação da Qualidade de Vida de Pacientes Submetidos à Cirurgia Cardíaca no Estado de Sergipe. Caderno de Graduação-Ciências Biológicas e da Saúde-UNIT. 2013. p. 113-124. Disponível em: https://periodicos.set.edu.br/cadernobiologicas/article/view/1006/541 Acesso em: 07 de junho de 2021.

TORRATI, Fernanda; DANTAS, Rosana. Circulação extracorpórea e complicações no período pós-operatório imediato de cirurgias cardíacas. Acta paulista de enfermagem. v.25.n. 3. São Paulo. 2012. Disponível em: https://doi.org/10.1590/S0103-21002012000300004 Acesso em: 07 de junho de 2021.

WOODS, Susan L.; FROELICHER, Erika S.; MOTZER, Sandra. U. Enfermagem em Cardiologia. 4. ed. São Paulo: Manole, 2005. p. 686-694.

Enviado: Junho, 2021.

Aprovado: Junho, 2021. 